\title{
Colonia Actia Nicopolis \\ Überlegungen anläßlich der Neuedition von CIL III 7334 in CIPh I 1, 78
}

\author{
Frank Daubner
}

Der marmorne Inschriftenstein des Decimus Furius Octavius ist (CIL III $7334=$ ILS 2080) seit langem bekannt. ${ }^{1}$ Bei der editio princeps von 1888 handelt es sich um einen Rekonstruktionsversuch P.-F. Foucarts anhand einer fehlerhaften Abschrift des Textes

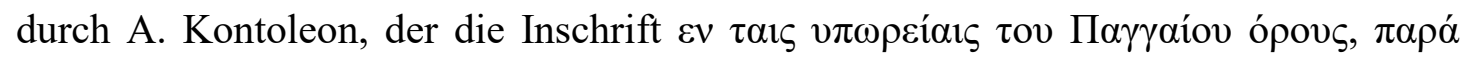

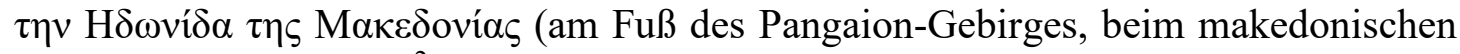
Idonia) gefunden hat. ${ }^{2}$ R. Cagnat hat im Jahr darauf einige Verbesserungen vorgeschlagen. ${ }^{3}$ M. Dimitsas hat den Text Foucarts und größtenteils auch dessen Kommentare in sein Corpus der makedonischen Inschriften übernommen. ${ }^{4}$ Im CIL von 1902 hat Th. Mommsen, weiterhin ohne Autopsie oder Abklatsch, einige weitere Konjekturen vorgenommen. Die Inschrift wird hier Serres zugewiesen, ${ }^{5}$ ein Fehler, der sich in fast allen folgenden wissenschaftlichen Beiträgen zur Inschrift wiederfindet. Ebenso wird im CIL suggeriert, der Text könne nach Z. 16, die in allen bisherigen Publikationen die letzte war, nach unten beliebig erweitert werden. Tatsächlich ist lediglich noch für eine Zeile Platz vorhanden, bevor der etwas erhabene Rand des Inschriftenfeldes und das untere Abschlußprofil anschließen, die auch nach dem Verlust der linken unteren Ecke weiterhin zu sehen waren. A. Salač hatte den Stein im Jahr 1921 noch in situ gesehen, allerdings fehlte zu dieser Zeit schon die linke untere Ecke. ${ }^{6}$ Wiederentdeckt wurde er in den späten 1960er Jahren von Ch. Koukouli. ${ }^{7}$

Die Lesung des Textes war nicht in jedem Punkt zuverlässig, da nie eine Autopsie durchgeführt wurde und die linke untere Ecke des Steins spätestens seit 1921 verschollen war. Bei Grabungen im Heiligtum des Heros Aulonites in Kipia ist sie im Jahr 1999 wiedergefunden worden, ${ }^{8}$ und 2014 hat C. Brélaz die vollständige

\footnotetext{
${ }^{1}$ Die vor 2014 letzte Edition mit ausführlicher Bibliographie: P. Pilhofer, Philippi Band 2. Katalog der Inschriften von Philippi, Tübingen ${ }^{2} 2009$ (im folgenden I.Philippi), 766-770, Nr. 617. Dort noch nicht berücksichtigt ist L. Ruscu, Actia Nicopolis, ZPE 157, 2006, 247-255, die S. 250 einen nicht überprüften Text der Inschrift gibt. Vgl. AE 2006, 1352. Der Name des Weihenden ist ergänzt nach AE 1975, 23 aus Rom: D(is) M(anibus) / Euphrosyno / servo / D(ecimus) Furius Octavius / Secundus / evocatus Aug(usti), vgl. E. Dąbrowa, Legio X Fretensis. A Prosopographical Study of its Officers (I-III c. A.D.), Stuttgart 1993, 91 Nr. 25. Ein unüberprüfter Text findet sich bei É. Guerber, La fondation de Nicopolis par Octavien: Affirmation de l'idéologie impériale et philhellénisme, in: A. Gangloff (Hg.), Lieux de mémoire en Orient grec à l'époque impériale, Bern 2013, 255-277; hier: 264 f.

${ }^{2}$ P.-F. Foucart, Inscription latine de Macédoine, BCH 12, 1888, 424-427.

${ }^{3}$ R. L. V. Cagnat, Inscription latine de Macédoine, BCH 13, 1889, $182 \mathrm{f}$.

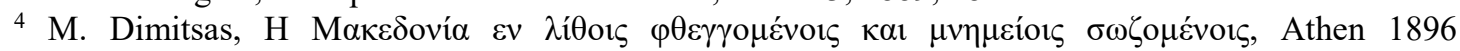
(Nachdruck Chicago 1980), Nr. 969.

${ }^{5}$ Mommsen schreibt: „Locus donec accuratus definietur, interim inter Serrhenses rettuli.“

${ }^{6}$ A. Salač, Inscriptions du Pangée, de la région Drama-Cavalla et de Philippes, BCH 47, 1923, 49-96; hier: $52 \mathrm{Nr}$. 5: „Elle se trouve actuellement sur un plateau dominant la route, entre les villages de Kodjani [heute Kipia] et Bostandji. Le marbre n'est plus complet, tout l'angle inférieur gauche ayant disparu." Salač hat einen Abklatsch anfertigen lassen, gibt allerdings keinen Text.

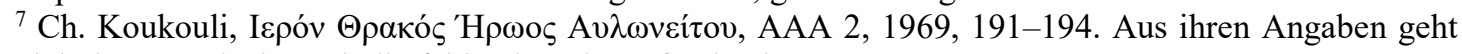
nicht hervor, ob sie auch die fehlende Ecke gefunden hat.

8 Der restaurierte Stein befindet sich im Garten des Museums von Kavalla und trägt die Inventarnummer $\Lambda$ 801; die wiedergefundene Ecke ist $\Lambda 1572$ (Stand 2010).
} 
und geprüfte Inschrift im ersten erschienenen Band der Inschriften von Philippi veröffentlicht. ${ }^{9}$ Hier soll vor allem untersucht werden, inwiefern die nun gesicherten Lesungen der Inschrift historisch relevant sind in Hinblick auf die Frage, ob das epirotische Nikopolis eine römische Kolonie war oder nicht.

Der folgende, am (bis auf die bisher unbekannte Z. 17) gut lesbaren Stein geprüfte und korrigierte Text zeigt ab Z. 5 je durch einen senkrechten Strich die Grenze des links abgebrochenen Teils an.

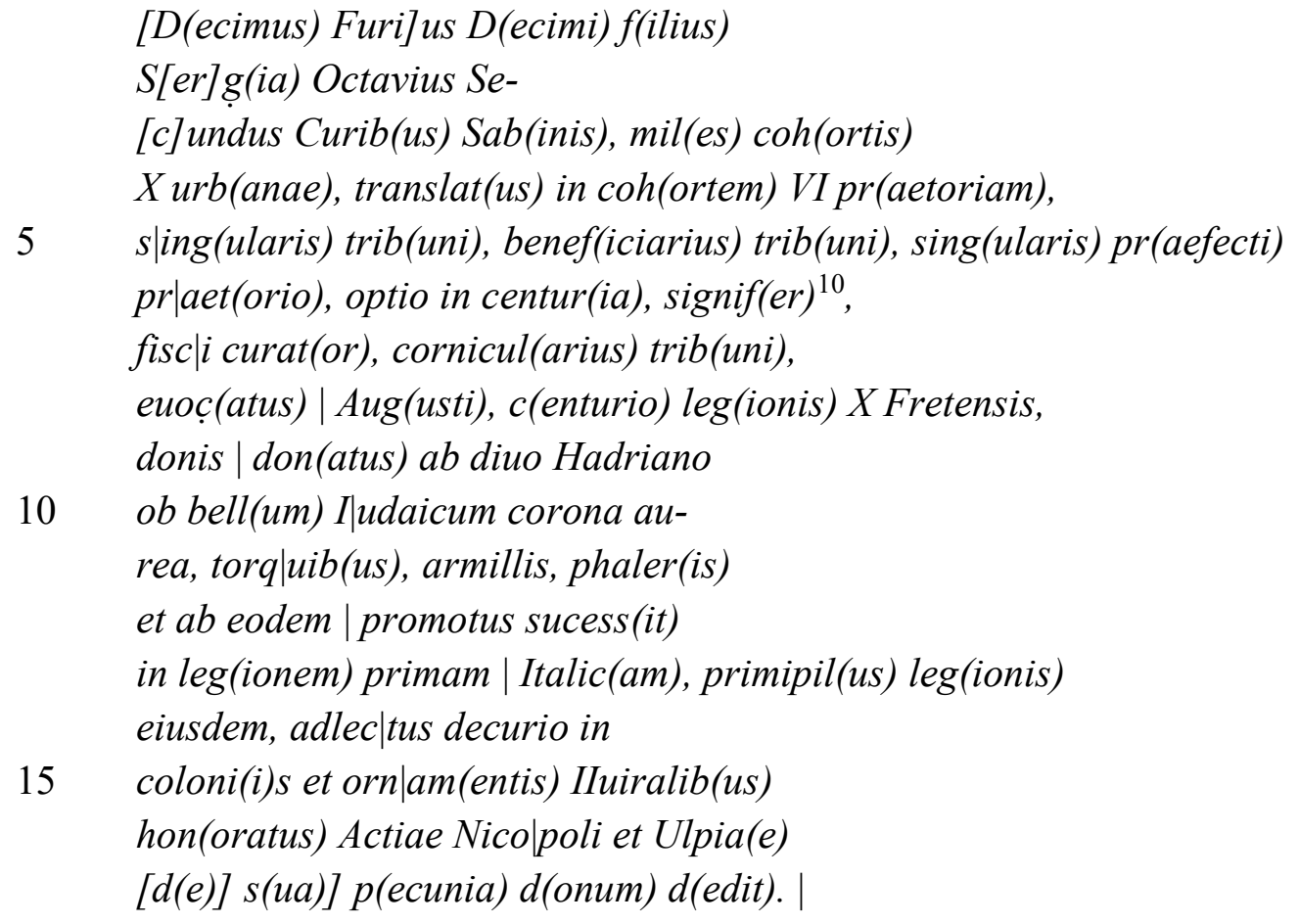

10 ob bell(um) I|udaicum corona au-

rea, torq|uib(us), armillis, phaler(is)

et ab eodem | promotus sucess(it)

in leg(ionem) primam | Italic(am), primipil(us) leg(ionis)

eiusdem, adlec|tus decurio in

15 coloni(i)s et orn|am(entis) Iluiralib(us)

hon(oratus) Actiae Nico|poli et Ulpia(e)

[d(e)] s(ua)] p(ecunia) d(onum) d(edit). |

„Decimus Furius Octavius Secundus, Sohn des Decimus, aus der Tribus Sergia, aus Cures Sabini, Soldat der zehnten städtischen Kohorte, versetzt in die sechste Prätorianerkohorte, singularis des Tribunen, beneficiarius des Tribunen, singularis des Prätorianerpräfekten, optio in der Zenturie, Feldzeichenträger, Kassenaufseher, cornicularius des Tribunen, evocatus des Kaisers, centurio der zehnten Legion Fretensis, ausgezeichnet vom göttlichen Hadrian aus Anlaß des Jüdischen Krieges mit einem goldenen Kranz, mit torques, mit Armspangen und mit phalerae und von demselben in die erste Legion Italica befördert, primipilus ebendieser Legion, zum decurio ernannt und mit duumviralen ornamenta geehrt in den Kolonien Actia Nicopolis und Ulpia, hat dies von seinem eigenen Gelde gestiftet.“

Die Inschrift ist bis auf ein eventuell fehlendes $i$ in Z. 15 und ein fehlendes e in Z. 16 fehlerfrei. Die letzte Zeile ist in kleineren Buchstaben gehalten; die Abkürzung steht nicht mittig auf dem Stein, sondern ist nach links versetzt, so daß sie ganz auf dem abgebrochenen Teil zu stehen kommt. Sie wurde von Kontoleon offensichtlich nicht gesehen oder nicht kopiert. Das erhaltene und erkennbare PDD könnte womöglich

\footnotetext{
${ }^{9}$ C. Brélaz, Corpus des inscriptions grecques et latines de Philippes. Tome II: La colonie romaine, partie 1: La vie publique de la colonie, Athen 2014, Nr. 78.

${ }^{10}$ Die NIF-Ligatur am Ende von Z. 6 hat Brélaz ignoriert.
} 
auch $\mathrm{zu}$...p (osuit) d(ecreto) d(ecurionum) oder ...p (ublice) d(ecreto) d(ecurionum) aufgelöst werden, was für eine Weihung in einem Heiligtum der colonia auch nicht ungewöhnlich wäre. ${ }^{11}$

Der Fundort der Inschrift, der sicher auch der Aufstellungsort war, ist mittlerweile als das zu Philippi gehörende Heiligtum des Heros Aulonites identifiziert worden. Dieser war eine lokale Variante des thrakischen Reitergottes. ${ }^{12}$ Der Kult dieses Heros war einer der bedeutendsten der römischen Kolonie Philippi. Er war neben dem Kaiserkult der einzige, von dem wir wissen, daß er offiziell von der colonia begangen wurde, und hatte einige weitere Besonderheiten. ${ }^{13}$ Den Aulonites verehrten die römischen, griechischen und thrakischen Bewohner des Territoriums der Stadt; im 3. Jh. n. Chr. findet er sich auf ihren Münzen, die ansonsten nie lokale Themen darstellen. ${ }^{14}$ Somit handelt es sich um eines der wichtigsten Heiligtümer Philippis, und die Inschrift des Furius Octavius reiht sich in die Weihungen in griechischer und lateinischer Sprache ein, die seitdem in seinem $20 \mathrm{~km}$ vom städtischen Zentrum entfernt liegenden Heiligtum gefunden wurden. Die Weihung (womöglich einer Statue, deren Basis der Inschriftenstein bildete) erfolgt ganz römisch. Der größte Teil des Inschriftenfeldes wird von der ausführlichen Schilderung des cursus honorum des Veteranen eingenommen, bevor womöglich ganz am Ende und kleiner als der Haupttext, vor allem auch viel kleiner als der in doppelter Buchstabengröße genannte Name des Weihenden, der Anlaß der Inschriftsetzung nachgetragen wird. ${ }^{15}$ Das wäre allerdings ungewöhnlich; meist wird am Anfang der Inschrift mitgeteilt, worauf sie sich bezieht.

Sicher ist nun auch, daß in Z. 16 tatsächlich vom epirotischen Nikopolis die Rede ist. Die Inschrift spielt keine prominente Rolle bei der Diskussion um den Status dieser augusteischen Gründung im Westen Griechenlands. Da das betreffende wichtige Stück des Texts fehlte, ${ }^{16}$ konnte man eine nicht unwahrscheinliche Fehllesung Kontoleons annehmen, denn darüber, daß Nikopolis keine römische Kolonie war, scheint im großen und ganzen Einigkeit bestanden zu haben. Zwar bezeichnen Tacitus

\footnotetext{
${ }^{11}$ Vgl. I.Philippi 132; 240; 281; 283; 451. Womöglich kann der Anfang der Zeile bei besserem Licht oder auf einem Abklatsch erkannt werden. Brélaz konnte jedenfalls nicht mehr lesen als ich und bevorzugt die Auflösung als [l(oco)] d(ato) p(ublice) d(ecreto) d(ecurionum). Das punktiert gegebene, als dato ergänzte $\mathrm{d}$ ist eher erraten als gesehen; ich habe eher ein s gesehen, das aber zu unsicher, als daß es punktiert gegeben werden sollte.

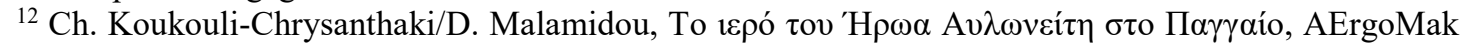
3, 1989, 553-567. Zur Lage vgl. A. Avraméa, Tabula Imperii Romani K 35, I: Philippi, Athen 1993, 31. Die Inschriften aus dem Heiligtum: I.Philippi Nr. 616-633; G. A. Pikoulas, H $\chi \omega ́ \rho \alpha \tau \omega v ~ \Pi 1 \varepsilon ́ \rho \omega v$.

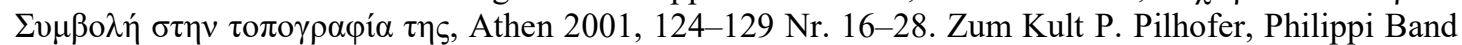
1. Die erste christliche Gemeinde Europas, Tübingen 1995, 93-100; Ch. Tsochos, Die Religion in der römischen Provinz Makedonien, Stuttgart 2012, 76-80; G. Falezza, I santuari della Macedonia in età romana. Persistenze e cambiamenti del paesaggio tra II secolo a.C. e IV secolo d.C., Rom 2012, 338344.

${ }^{13}$ I.Philippi Nr. 629a: Pro salute coloniae Iuliae Aug(ustae) Philippensis auf einem Altar.

${ }_{14}$ M. Amandry, Le monnayage de la Res Publica Coloniae Philippensium, in: U. Peter (Hg.), Stephanos numismatikos. Edith Schönert-Geiss zum 65. Geburtstag, Berlin 1998, 23-31.

${ }^{15} \mathrm{Zu}$ diesem und ähnlichen Phänomenen W. Eck, There are no cursus honorum Inscriptions. The Function of the cursus honorum in Epigraphic Communication, SCI 28, 2009, 79-92; bes. 82-84.

${ }^{16}$ Das ist durchaus aufgefallen; siehe D. Strauch, Römische Politik und griechische Tradition. Die Umgestaltung Nordwest-Griechenlands unter römischer Herrschaft, München 1996, 168 Anm. 54; Ruscu (wie Anm. 1) 250 f.; C. H. Lange, Res Publica Constituta. Actium, Apollo and the Accomplishment of the Triumviral Assignment, Leiden 2009, 101.
} 
und Plinius die Stadt bzw. Actium als colonia, ${ }^{17}$ doch erwähnen die übrigen, vor allem sämtliche griechischen Quellen nichts dergleichen. Auch im archäologischen Befund deutet nichts auf eine Kolonie, und die Münzen sind ganz griechisch, ebenso die Institutionen, die Inschriften und die Namen. ${ }^{18}$ Nun ist colonia ein Rechtstitel, den ein Gemeinwesen entweder hat oder nicht hat, und da es keine Anzeichen dafür in den Münzen und Inschriften gibt, galt Nikopolis der communis opinio offensichtlich nicht als colonia. ${ }^{19}$ Es wird, ausgehend von Plinius' anscheinender Erwähnung zweier unterschiedlicher Gemeinwesen, die Möglichkeit diskutiert, es habe sich um eine Doppelgemeinde, also um nebeneinander existierende Gemeinden unterschiedlichen Rechtsstatus' - eine römische colonia neben einer griechischen polis - gehandelt. ${ }^{20}$ Der Beitrag der Inschrift wäre gewichtig, und es ist doch bei einer so detaillierten Inschrift unwahrscheinlich, daß mit der Formulierung adlectus decurio in coloni $(i)$ s et ornam(entis) IIviralib(us) don(atus) Actiae Nicopoli et Ulpia(e) gemeint sein kann, daß Furius Octavius in einigen nicht näher genannten Kolonien zum Dekurionen ernannt wurde, während ihn die beiden Städte Aktia Nikopolis und Ulpia mit den ornamenta eines Duumvirn geehrt haben. ${ }^{21}$ Abgesehen davon, daß es ungewöhnlich

17 Tac. ann. 5, 10, 3 f.: Nicopolis romana colonia; Plin. nat. 4, 1, 4 f.: in ore ipso colonia Augusti Actium cum templo Apollinis nobile ac civitate libera Nicopolitana.

${ }^{18}$ Quellen zu Nikopolis als griechische Polis: Suet. Aug. 18, 2; Strab. 7, 7, 5; Paus. 5, 23, 3; Cass. Dio 51, 1, 3; Antipatros von Thessaloniki(?) Anth. Pal. 9, 553. Zum griechischen Charakter des archäologischen Befundes: S. J. Ellis Jones, Cities of Victory - Patterns and Parallels, in: E. Chrysos (Hg.), Nicopolis I. Proceedings of the First International Symposium on Nicopolis, Preveza 1987, 99108. Münzprägung: D. Calomino, Nicopolis d'Epiro. Nuovi studi sulla zecca e sulla produzione monetale, Oxford 2011; ders., Actia Nicopolis. Coinage, Currency and Civic Identity, in: F. López Sánchez (Hg.), The City and the Coin in the Ancient and Medieval Worlds, Oxford 2012, 103-116.

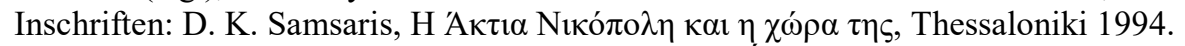

${ }^{19}$ Zur Diskussion Th. Ch. Sarikakis, Nicopolis d'Épire était-elle une colonie romaine ou une ville grecque?, Balkan Studies 11, 1970, 91-96; K. L. Zachos/E. A. Pavlidis, Nikopolis, die Siegesstadt bei Actium. Die Forschungsergebnisse der letzten Jahre, in: H. Frielinghaus/J. Stroszeck (Hgg.), Neue Funde und Forschungen $\mathrm{zu}$ griechischen Städten und Heiligtümern. Festschrift für Burkhardt Wesenberg zum 65. Geburtstag, Möhnesee 2010, 139-160; É. Guerber, La permanence et les limites de la liberté des cités du monde hellénophone sous le Haut-Empire romain, in: C. Feyel u. a. (Hgg.), Communautés locales et pouvoir central dans l'Orient hellénistique et romain, Paris 2012, 451-497; hier: 472-474; R. Haensch, Capita provinciarum. Statthaltersitze und Provinzialverwaltung in der römischen Kaiserzeit, Mainz 1997, 328 mit Anm. 36; U. Kahrstedt, Die Territorien von Patrai und Nikopolis in der Kaiserzeit, Historia 4, 1950, 549-561; hier: 559 f.; Lange (wie Anm. 16) 100-106.

${ }^{20}$ N. Purcell, The Nicopolitan Synoecism and Roman Urban Policy, in: E. Chrysos (Hg.), Nicopolis I. Proceedings of the First International Symposium on Nicopolis, Preveza 1987, 71-90; Ruscu (wie Anm. 1); Guerber (wie Anm. 1) 263-267. Literatur zu Doppelgemeinden bei Strauch (wie Anm. 16) 189 f. Zu hellenistischen Doppelgemeinden vgl. A. Ivantchik, Три надписи фиасов эллинистического времени из Танаиса. Новые данные о греко-иранском взаимодействии в Танаисе дополемоновской эпохи, VDI 2008/2, 57-72; zu caesarisch-augusteischen Doppelgemeinden in Kleinasien T. Esch, Zur Frage der sogenannten Doppelgemeinden. Die caesarische und augusteische Kolonisation in Kleinasien, in: E. Winter (Hg.), Vom Euphrat bis zum Bosporos. Kleinasien in der Antike. Festschrift für Elmar Schwertheim zum 65. Geburtstag Bd 1, Bonn 2008, 199-216.

${ }^{21}$ So P. Pilhofer in der in I.Philippi S. 768 gegebenen Übersetzung. Kahrstedt (wie Anm. 19) 560 möchte mit einer kaum nachvollziehbaren Argumentation das colonia nur auf Ulpia beziehen, das er wohl irrtümlich für den Fundort der Inschrift und damit den Wohnort des Octavius hält. (Zum Beinamen Ulpia s. B. Galsterer-Kröll, Untersuchungen zu den Beinamen der Städte im Imperium Romanum, Epigraphische Studien 9, 1972, 44-145; hier: 50; 54; 76 f.) Daß ein Veteran von mehreren Städten, ob Kolonien oder Poleis, geehrt wurde, ist nicht ungewöhnlich; vgl. CIL III $753=7429$ und CIL III 14416 = ILS 7178 aus Ulpia Oescus sowie ILS 6933, 6943 und 7035, alle für M. Pompeius Lucius, bf. cos. leg. I Italicae. 
wäre, die Kolonien, in denen Octavius geehrt wurde, nicht zu nennen, ist es kaum vorstellbar, daß Städte, die keine Kolonien sind, duumvirale ornamenta verleihen können. Die wieder aufgefundene linke untere Ecke des Steins bestätigt also zum einen die erste Lesung Kontoleons, zum anderen trägt sie nichts zur Klärung der Frage bei, welches Ulpia gemeint ist. ${ }^{22}$ Vor allem aber zeigt die nunmehr gesicherte Lesung Actiae Nicopoli, daß Nikopolis zumindest in hadrianischer Zeit eine römische colonia oder eine Doppelgemeinde gewesen sein muß. So muß wohl auch dem sorgfältigen Tacitus geglaubt werden, Nikopolis als romana colonia bezeichnet. Das geschieht im Rahmen der Episode, als C. Poppaeus Sabinus, cos. 9 n. Chr., im Jahre 30/31 als Statthalter von Macedonia und Achaea $^{23}$ den falschen Drusus sucht, der auf den Kykladen gesehen worden sein soll. ${ }^{24}$ Eine Koloniegründung durch Tiberius ist ausgeschlossen, so daß die Kolonie Nikopolis durch Augustus gegründet worden sein muß. Die konkrete Ausgestaltung dieser Gründung bleibt zu untersuchen.

\title{
Kontakt zum Autor:
}

\author{
Frank Daubner \\ Universität Trier \\ Fachbereich III - Alte Geschichte \\ Email: daubner@uni-trier.de
}

\footnotetext{
${ }^{22}$ Darüber kann man nur Vermutungen anstellen. Daß sich der Name parallel zu Actia ebenfalls auf Nicopolis beziehen ließe, wäre in dieser Wortstellung ungewöhnlich. In dem Fall kämen jedoch Nikopolis am Nestos/Mestos und Nikopolis beim Istros in Frage. Ein Beiname war sicher nicht vorhanden, auch wenn Brélaz vermutet, ein solcher könne am Beginn von Z. 17 gestanden haben. Das ist unwahrscheinlich, da im freigelassenen Bereich am Ende von Z. 16 noch Platz für mindestens zwei Buchstaben ist, am Beginn von Z. 17 Platz für höchstens vier, und Z. 17 mit dem Setzungsvermerk in viel kleineren Buchstaben gehalten ist. So muß es eine Stadt gewesen sein, die entweder in der Nähe lag oder die dem Octavius so selbstverständlich war, daß er glaubte, nicht spezifischer werden zu müssen. Im ersteren Fall wären Nikopolis am Nestos/Mestos, Topeiros oder Serdika naheliegend, die allerdings keine Kolonien waren, im letzteren eher Oiskos als die zumeist angenommene Kolonie Sarmizegetusa: Die legio I Italica, in der er als Centurio diente, lag seit Vespasian in Novae am Donaulimes; in der Nähe wurde von Trajan die Veteranenkolonie Ulpia Oescensium angelegt (CIL III 7429; V. Velkov, Развитие на градския живот. Пътна система [История на България 1], Sofia 1979, 307), die einige Offiziere der legio I geehrt hat, wie die in der vorigen Anmerkung genannten Inschriften zeigen. Vgl. E. Ritterling, Legio (I Italica), RE XII 2, 1925, 1407-1417, und T. Sarnowski, Primi ordines et centuriones legionis I Italicae und eine Dedikation an Septimius Severus aus Novae in Niedermoesien, ZPE 95, 1993, 205-219. In zwei Inschriften aus Ulpia Nicopolis ad Istrum, in dessen griechischen öffentlichen Inschriften stets Rat und Volk agieren (ebenso wie in IGBulg 2335, der einzigen Inschrift aus Ulpia Nicopolis ad Mestum, in der Magistrate auftauchen), sind Aedilen genannt: CIL III 12435 = IGBulg 391 und in AE 1991, 1376; aus der Stadt stammen einige Legionäre und Prätorianer, letztere allerdings erst nach der Constitutio Antoniniana. Das ist freilich nicht hinreichend, um hier eine Kolonie oder ein Municipium zu postulieren, jedoch bleibt $\mathrm{zu}$ fragen, ob die vier Veteranen der tribus Sergia, die als Heimat ein Nicopolis angeben und die von Ruscu (wie Anm. 1) 253 f. dem epirotischen Nikopolis zugeschlagen werden, tatsächlich dorther kommen müssen, zumal die Steine aus Bath, Rom, Pannonien und Untermoesien stammen (CIL VII 51, CIL VI 375 = ILS 2104, AE 1914, 101, CIL III 6144 = IGBulg 376). Lange (wie Anm. 16) 101, möchte sie zwar keinesfalls Nicopolis ad Istrum zuordnen, da ihn die tribus Sergia an die augusteische Gründung Antiocheia in Pisidien erinnere, deren Kolonisten ebenfalls in die Sergia eingeschrieben waren, doch verliert das Argument an Überzeugungskraft, wenn man berücksichtigt, daß zwei Nikopolitaner bekannt sind, die der tribus Papiria angehörten: CIL III 1481 aus dem dakischen Apulum und CIL III $12397=$ IGBulg 147 aus Untermoesien.

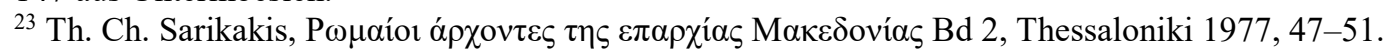

${ }^{24}$ Tac. ann. 5, 10, $3 \mathrm{f}$.
} 